\title{
A generalized anisotropic quadric yield criterion and its application to bone tissue at multiple length scales
}

\author{
J. J. Schwiedrzik · U. Wolfram • P. K. Zysset
}

Received: 5 September 2012 / Accepted: 9 January 2013 / Published online: 15 February 2013

(C) Springer-Verlag Berlin Heidelberg 2013

\begin{abstract}
Nonlinear computational analysis of materials showing elasto-plasticity or damage relies on knowledge of their yield behavior and strengths under complex stress states. In this work, a generalized anisotropic quadric yield criterion is proposed that is homogeneous of degree one and takes a convex quadric shape with a smooth transition from ellipsoidal to cylindrical or conical surfaces. If in the case of material identification, the shape of the yield function is not known a priori, a minimization using the quadric criterion will result in the optimal shape among the convex quadrics. The convexity limits of the criterion and the transition points between the different shapes are identified. Several special cases of the criterion for distinct material symmetries such as isotropy, cubic symmetry, fabric-based orthotropy and general orthotropy are presented and discussed. The generality of the formulation is demonstrated by showing its degeneration to several classical yield surfaces like the von Mises, Drucker-Prager, Tsai-Wu, Liu, generalized Hill and classical Hill criteria under appropriate conditions. Applicability of the formulation for micromechanical analyses was shown by transformation of a criterion for porous cohesive-frictional materials by Maghous et al. In order to demonstrate the advantages of the generalized formulation, bone is chosen as an example material, since it features yield envelopes with different shapes depending on the considered length scale. A fabric- and density-based quadric criterion for the description of homogenized material behavior of trabecular bone
\end{abstract}

Electronic supplementary material The online version of this article (doi:10.1007/s10237-013-0472-5) contains supplementary material, which is available to authorized users.

J. J. Schwiedrzik (凶) · U. Wolfram · P. K. Zysset

Institute of Surgical Technology and Biomechanics, University of Bern, Stauffacherstr. 78, 3014 Bern, Switzerland

e-mail: jakob.schwiedrzik@istb.unibe.ch is identified from uniaxial, multiaxial and torsional experimental data. Also, a fabric- and density-based Tsai-Wu yield criterion for homogenized trabecular bone from in silico data is converted to an equivalent quadric criterion by introduction of a transformation of the interaction parameters. Finally, a quadric yield criterion for lamellar bone at the microscale is identified from a nanoindentation study reported in the literature, thus demonstrating the applicability of the generalized formulation to the description of the yield envelope of bone at multiple length scales.

Keywords Yield criterion - Strength · Plasticity · Bone $\cdot$ Porosity

\section{Introduction}

Nonlinear computational analysis of materials showing elasto-plasticity or damage behavior relies on knowledge of their yield behavior and strengths under complex stress states. The yield or failure criterion of the material is usually described by a convex function taking into account the influence of the shear and normal stresses as well as their interactions. Many criteria have been proposed in the past like the von Mises (1913) or Hill (1951) criteria for plastically incompressible materials, especially metals, or the DruckerPrager criterion for dilatant cohesive-frictional materials (Prager and Drucker 1952). These functions, although of phenomenological nature, have proven to be valuable tools in mechanics of irreversible processes, as they can often be used as closed-form approximations of the complex material strength behavior. The criteria are formulated as explicit functions of the components of the stress tensor and a set of material constants that may be identified by independent tests. When solving mechanical problems using numerical 
methods, it is often desirable to use smooth functions as yield surfaces as standard backprojection procedures may be used in this case (Curnier 1994). However, some classical criteria like the one proposed by Prager and Drucker (1952) feature singular points where the normal on the yield surface is non-unique. In this case, it is often desirable to use smooth approximations of the actual yield function in order to avoid the implementation of special algorithms and/or numerical instabilities. In the framework of computational mechanics, strength criteria are frequently combined with dissipation mechanisms like damage or plasticity, that is, the accumulation of inelastic strains. These formulations often make use of hardening mechanisms such as dilatation and translation of the yield surface as a function of scalar or tensorial hardening variables. In particular, in the case of isotropic hardening, the use of functions that are homogeneous of degree one as a yield criterion is beneficial, since it simplifies the implementation of numerical algorithms like implicit backprojections.

One obstacle when dealing with elasto-plasticity in a computational mechanics framework is the choice of the correct yield surface. Since the formulations are usually valid for a certain group of materials and not of a general nature, care must be taken to identify the right one for the given application. This is especially the case when strength data are available for given load directions and a yield function needs to be identified by means of minimization procedures. In this sense, it would be desirable to use a formulation that describes a wide range of materials while retaining a simple and explicit form in order to obtain the optimal shape of the yield surface with respect to the data points without severe restrictions due to the formulation used.

One class of surfaces that combines simplicity of the underlying function with the ability to take a large variety of shapes is the set of convex quadrics. It spans from parallel planes to ellipsoids, paraboloids, (half) hyperboloids of two sheets, cylinders and cones, which are popular choices for yield functions of materials like metals, geomaterials, porous foams and bone. In particular, in the case of bone tissue, a general formulation is desirable. Its yield envelope changes its shape drastically depending on the length scale considered and has been approximated by quadric surfaces in the past (Cowin 1986, 1989; Carnelli et al. 2011; Wolfram et al. 2012). Therefore, it was chosen as an example material in this work.

Bone is a hierarchical material with collagen, mineral and water as well as non-collagenous proteins (Rho et al. 1998; Fratzl and Weinkamer 2007; Wang and Gupta 2011) as the main constituents. The collagen molecules self-assemble into fibrils which are periodically reinforced by mineral platelets (Weiner and Wagner 1998; Fratzl and Weinkamer 2007). Empty pore space is filled with water and non-collagenous proteins. This basic unit then forms bundles of parallel mineralized fibrils embedded in an extra-fibrillar mineral matrix with a foam-like structure (Hellmich and Ulm 2002; Reisinger et al. 2010) that are called fibril arrays. There is a considerable amount of porosity present on every hierarchical level from the nano- to the macro-scale (Smith et al. 2008). Tai et al. (2006) showed some evidence that bone behaves like a cohesive-frictional material at the nanolevel due to its nanogranular structure. They suggested that increased yield properties in compression compared to tension reported in the literature (Yeni et al. 2004) may be explained by nanogranular friction between mineral particles and cohesion originating from the organic phase. Finite element simulations using plasticity and damage models featuring a conical yield surface were able to capture some of the characteristics reported from nanoindentation experiments on bone (Tai et al. 2006; Carnelli et al. 2010, 2011). Micromechanical considerations by Maghous et al. (2009) showed that the introduction of porosity into a cohesive-frictional material leads to a decrease in uniaxial strength properties as well as a change of the shape of the criterion from a conical to an eccentric elliptical surface.

When looking at bone at the macroscale, one distinguishes between two types of bone, that is, compact and trabecular. In compact bone, parallel fibril arrays form lamellae. These lamellae are arranged in layers around blood vessels (Haversian channels) forming cylindrical structures called osteons. Arrays of parallel osteons connected by interstitial bone regions make up the cortical bone structure. Cortical bone features microporosity of up to $10 \%$, mainly due to an interconnected network of cells and cell processes, the so called lacunar-canalicular network (Kristic 1991; Weiner and Wagner 1998). On the macroscopic level, it has been proposed by Cowin $(1979,1986,1989)$ in the past that an eccentric elliptical, that is, a Tsai-Wu yield surface is suitable for cortical bone.

Trabecular bone is an anisotropic and highly porous mineralized tissue enclosed in compact bone in the cores of flat and small bones and the epiphyses of long bones (Kristic 1991). Plate- and rod-like structures of bone form an open-cell foam (Gibson 1985). Large efforts have been undertaken in the past to identify the mechanical properties and morphology of this highly heterogeneous tissue and their underlying relationships (Keaveny et al. 2001; Zysset 2003; Gupta and Zioupos 2008). Historically, morphological parameters like volume fraction, surface density, trabecular thickness, spacing and number have been identified using quantitative stereology (Parfitt 1984). In order to assess the anisotropy of the trabecular structure, the mean intercept length (MIL) method was introduced by Whitehouse (1974). Within the last 20 years, the investigation into trabecular morphology has been facilitated by the development of new technologies like micro-computed tomography $(\mu \mathrm{CT})$. Hildebrand et al. (1999) proposed a methodology to measure 
morphological parameters directly from 3D $\mu \mathrm{CT}$ data. The three-dimensional distribution function of trabecular orientations is then mathematically modeled by means of a secondorder fabric tensor (Kanatani 1984; Harrigan and Mann 1984).

Cowin first established a relationship between volume fraction, fabric tensor and the homogenized anisotropic elasticity tensor and strength criterion for highly porous materials such as trabecular bone (Cowin 1985, 1986, 1989). An alternative model for anisotropic elasticity of cancellous bone based on fabric tensors was proposed by Zysset and Curnier (1995), which reduced the number of material parameters and a priori satisfied thermodynamic admissibility. For further information on fabric-based models, see for example, the review by Zysset (2003). The model was extended toward yield and strength properties leading to the introduction of a halfspace-wise defined fabric- and density-based generalized Hill criterion (Zysset and Rincón 2006), which was later identified by Rincón-Kohli and Zysset (2009). Arramon et al. (2000) proposed an anisotropic criterion for bone in stress space based on Kelvin modes, while other authors introduced strain-based formulations (Bayraktar et al. 2004; Cowin and He 2005). Recently, a fabric- and density-based Tsai-Wu criterion has been proposed and successfully identified for trabecular bone using nonlinear $\mu \mathrm{FE}$ and homogenization techniques (Wolfram et al. 2012).

As part of this paper, the quadric yield criterion will be presented in a general anisotropic formulation and special cases of material symmetry will be discussed. This includes the case of a fabric- and density-based orthotropic formulation that has been proven useful in the past in the description of anisotropic mechanical behavior of bone (Zysset and Rincón 2006; Wolfram et al. 2012). Additionally, it will be shown that material properties may be obtained from a known TsaiWu criterion by means of a straightforward transformation of the interaction coefficients. The generality of the formulation will be demonstrated by showing that the quadric criterion degenerates to several classical yield criteria including the ones of Drucker and Prager (1952), von Mises (1913), Tsai and $\mathrm{Wu}$ (1971), Liu et al. (1997) and Hill (1951) when the right conditions are met. Applicability of the formulation for micromechanical analyses will be demonstrated by identification of a quadric yield surface from a criterion for porous cohesive-frictional materials (Maghous et al. 2009), which is also applicable for the description of bone at the microscale as a cohesive-frictional material (Tai et al. 2006) in interaction with nanoporosity. Finally, the suitability of the formulation to describe the yield surface of bone at several length scales will be presented by identification of material constants for criteria of bovine lamellar bone at the microscale as well as homogenized human trabecular bone at the macroscale.

\section{General formulation}

In the notation used in the following sections, scalars are written as $X$, vectors as $\boldsymbol{x}$, second-order tensors as $\boldsymbol{X}$, fourth-order tensors as $\mathbb{X}$, and tensor spaces as $\mathcal{X}$. The sign ':' denotes the double-contraction operation. In case of compositions of two fourth-order tensors $\left(\mathbb{X}_{i j k l}=\mathbb{Y}_{i j m n} \mathbb{Z}_{m n k l}\right)$ and of transformations of a second-order tensor with a fourth-order tensor $\left(\boldsymbol{X}_{i j}=\mathbb{Y}_{i j k l} \boldsymbol{Z}_{k l}\right)$, the sign ':' is not written. The operator $\otimes$ denotes the dyadic product $\mathbb{X}_{i j k l}=\boldsymbol{Y}_{i j} \boldsymbol{Z}_{k l}, \underline{\otimes}$ the tensorial product $\mathbb{X}_{i j k l}=\boldsymbol{Y}_{i k} \boldsymbol{Z}_{j l}$, and $\underline{\bar{\otimes}}$ the symmetric product $\mathbb{X}_{i j k l}=\frac{1}{2}\left(\boldsymbol{Y}_{i k} \boldsymbol{Z}_{j l}+\boldsymbol{Y}_{i l} \boldsymbol{Z}_{j k}\right)$.

We postulate an anisotropic quadric yield criterion in stress space $\mathcal{S}$ :

$Y(S):=\sqrt{\boldsymbol{S}: \mathbb{F} \boldsymbol{S}}+\boldsymbol{F}: \boldsymbol{S}-1=0$

It contains a fourth-order tensor $\mathbb{F}$ and a second-order tensor $\boldsymbol{F}$ defining the origin, orientation and shape of the criterion. Its surface normal is given by:

$\nabla_{\boldsymbol{S}} Y=(\boldsymbol{S}: \mathbb{F} \boldsymbol{S})^{-\frac{1}{2}} \mathbb{F} \boldsymbol{S}+\boldsymbol{F}$

See Fig. 1 for a visualization of the possible shapes of the criterion. It is a homogeneous function of degree one $(Y(\lambda \boldsymbol{S})=\lambda Y(S), \quad \forall \lambda \in \mathbb{R})$, which allows for a straightforward introduction of isotropic hardening, that is, a dilatation of the yield surface with respect to the origin of stress space, in the form of

$Y(\boldsymbol{S}, \kappa):=\sqrt{\boldsymbol{S}: \mathbb{F} \boldsymbol{S}}+\boldsymbol{F}: \boldsymbol{S}-r(\kappa)=0$

with an isotropic hardening function $r(\kappa)$ of a scalar hardening variable $\kappa$. For tensorial hardening with a tensor function $\mathbb{K}(\kappa)$, the criterion takes the form

$Y(S, \kappa):=\sqrt{S: \mathbb{K}(\kappa) \mathbb{F} \mathbb{K}(\kappa) S}+\mathbb{K}(\kappa) \boldsymbol{F}: S-1=0$,

where $\mathbb{K}(\kappa)$ features the same symmetries as $\mathbb{F}$. In order for (1) to be real, the fourth-order tensor $\mathbb{F}$ needs to be positive semidefinite, which may be expressed as:

$S: \mathbb{F} S \geq 0, \quad \forall S \in \mathcal{S}$

$\mathbb{F}$ has major symmetry $\left(\mathbb{F}_{i j k l}=\mathbb{F}_{k l i j}\right)$ and both minor symmetries $\left(\mathbb{F}_{i j k l}=\mathbb{F}_{j i k l}=\mathbb{F}_{i j l k}=\mathbb{F}_{j i l k}\right)$, which allows to project the associated space defined on $\left(\mathcal{R}^{3} \times \mathcal{R}^{3}\right) \times\left(\mathcal{R}^{3} \times\right.$ $\mathcal{R}^{3}$ ) onto a symmetric matrix space defined on $\mathcal{R}^{6} \times \mathcal{R}^{6}$. This is a standard procedure in continuum mechanics (Mehrabadi and Cowin 1990; Zysset and Curnier 1995) and simplifies the mathematical discussion of the problem. In this case, positive semidefiniteness of the fourth-order tensor $\mathbb{F}$ is given if the determinants of all principal minors of its projection onto the symmetric matrix space are positive. Positive semidefiniteness as defined in (5) requires that

$1-\boldsymbol{F}: S \geq 0$ 
(a)

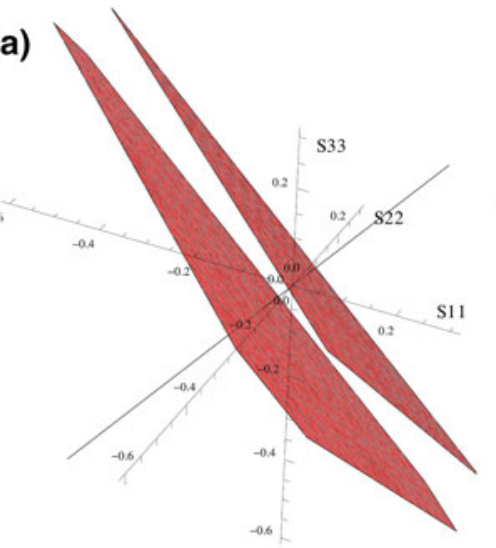

(b)

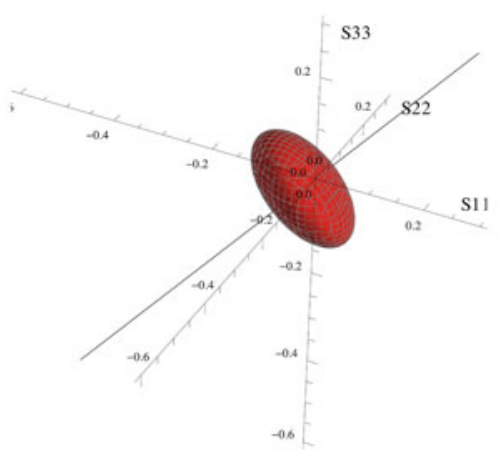

(d)

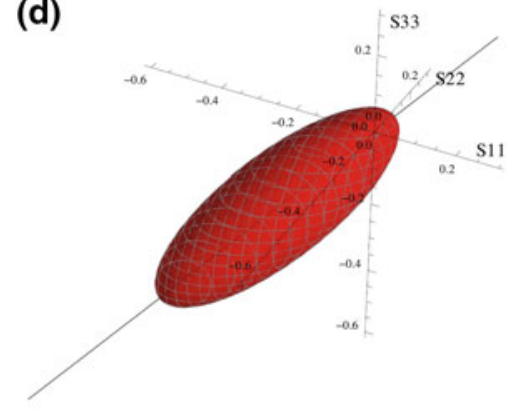

(g)

(e)

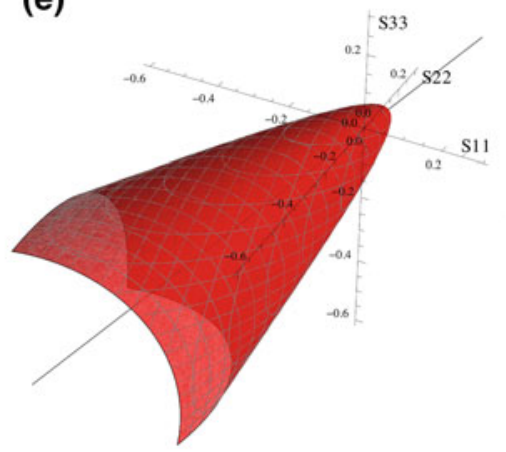

(c)

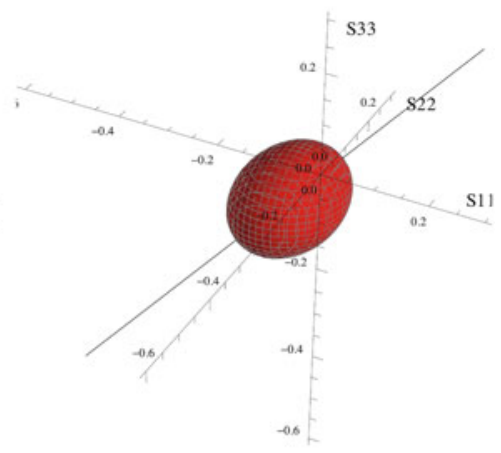

(f)

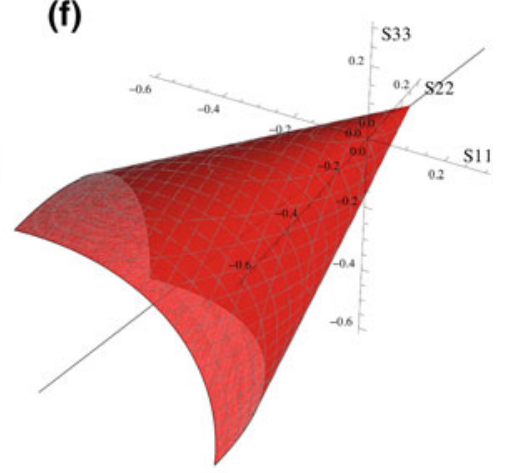

Fig. 1 Shape of the isotropic quadric yield surface in normal stress space for a $\zeta_{0}=-1$, b $\zeta_{0}=-0.5$, c $\zeta_{0}=0.0$, d $0<\zeta_{0}<\zeta_{0}$,crit, e $\zeta_{0, \text { crit }}<\zeta_{0}<0.5$, and $\mathbf{f} \zeta_{0}=0.5$ as well as $\mathbf{g}$ in shear stress space

which defines a half-hyperspace, in order for (1) to hold true. In order for (1) to describe a convex surface, its Hessian needs to be positive semidefinite, that is,

$S: \nabla_{S}^{2} Y S \geq 0, \quad \forall S \in \mathcal{S}$

with the Hessian

$\nabla_{S}^{2} Y=(\boldsymbol{S}: \mathbb{F} \boldsymbol{S})^{-\frac{1}{2}} \mathbb{F}-(\boldsymbol{S}: \mathbb{F} \boldsymbol{S})^{-\frac{3}{2}} \mathbb{F} \boldsymbol{S} \otimes \mathbb{F} \boldsymbol{S}$.

Substituting (8) into (7) leads to the requirement

$\sqrt{S: \mathbb{F} S}-\sqrt{S: \mathbb{F} S} \geq 0$,

which is true for all $S$ in $\mathcal{S}$ and any positive semidefinite $\mathbb{F}$. Therefore, positive semidefiniteness of the fourth-order tensor $\mathbb{F}$ as required in (5) is a necessary and sufficient requirement for the convexity of the criterion (1). If the requirements (5) and (6) are met, (1) is convex and may be squared without loss of information.

$$
\begin{aligned}
& \boldsymbol{S}: \mathbb{F} \boldsymbol{S}-(\boldsymbol{F}: \boldsymbol{S})^{2}+2 \boldsymbol{F}: \boldsymbol{S}-1=0, \\
& \quad(\boldsymbol{F}: \boldsymbol{S})^{2}=\boldsymbol{S}:(\boldsymbol{F} \otimes \boldsymbol{F}) \boldsymbol{S} .
\end{aligned}
$$

The mathematical discussion of the criterion within the boundaries given by (5) and (6) is therefore equivalent to the discussion of the quadratic form

$\boldsymbol{S}:(\mathbb{F}-\boldsymbol{F} \otimes \boldsymbol{F}) \boldsymbol{S}+2 \boldsymbol{F}: \boldsymbol{S}-1=0$

within the same boundaries. In order to find the midpoint of the criterion, the quadratic form (11) may be rewritten as proposed by Shih and Lee (1978): 
$(\boldsymbol{S}-\boldsymbol{A}): \mathbb{A}(\boldsymbol{S}-\boldsymbol{A})-1=0$

with a fourth-order tensor $\mathbb{A}$ describing the shape and orientation

$\mathbb{A}=\frac{\mathbb{F}-\mathbf{F} \otimes \mathbf{F}}{1+\mathbf{A}:(\mathbb{F}-\mathbf{F} \otimes \mathbf{F}) \mathbf{A}}$

and the second-order tensor $\boldsymbol{A}$ describing the shift of the quadric with respect of the origin of $\mathcal{S}$,

$\boldsymbol{A}=-(\mathbb{F}-\boldsymbol{F} \otimes \boldsymbol{F})^{-1} \boldsymbol{F}$.

The shape of the criterion is determined by the nature of the eigenvalues of the fourth-order tensor $\mathbb{F}-\boldsymbol{F} \otimes \boldsymbol{F}$. If all six eigenvalues are positive, the quadratic form (11) takes an hyperellipsoidal shape. When the eigenvalue whose eigentensor points in the main direction of the quadric vanishes, it becomes a hyperparaboloid. If this eigenvalue becomes negative and the others stay positive, $\mathbb{F}-\boldsymbol{F} \otimes \boldsymbol{F}$ becomes negative definite and the shift $\boldsymbol{A}$ changes its sign. In this case, the quadratic form (11) describes an elliptical hyperboloid of two sheets with the extreme case of a double-hypercone. The negative eigenvalue results from the ambiguity of the quadratic form (11) and does not lead to a loss of convexity of the criterion (1), as it is defined in the half-hyperspace (6) only. The boundary hyperplane of (6) separates the hyperboloid of two sheets described by (11) into two halfspace-wise convex surfaces. The criterion (1) then takes a hyperboloidal or hyperconical shape. The transition from hyperellipsoidal to hyperboloidal shape is a hyperparaboloid and may be found by requiring

$\operatorname{Det}(\mathbb{F}-\boldsymbol{F} \otimes \boldsymbol{F})=0$.

The multidimensional determinant of a fourth-order tensor may be evaluated as shown in, for example, Gelfand and Kapranov (1994). However, the calculations may be largely simplified by requiring the determinant of the projection of $\mathbb{F}-\boldsymbol{F} \otimes \boldsymbol{F}$ onto the symmetric matrix space defined on $\mathcal{R}^{6} \times \mathcal{R}^{6}$ to vanish. The hypercone is a limit case of the possible convex shapes of the criterion that is found when requiring

$\operatorname{Det}(\mathbb{F})=0$.

Several special cases of material symmetry ranging from isotropy to orthotropy will be discussed in the following subsections.

\subsection{Isotropy}

In the case of isotropy, the tensors $\mathbb{F}$ and $\boldsymbol{F}$ defining (1) are given by

$\mathbb{F}=-\zeta_{0} F_{0}^{2}(\boldsymbol{I} \otimes \boldsymbol{I})+\left(\zeta_{0}+1\right) F_{0}^{2}(\boldsymbol{I} \underline{\bar{\otimes}} \boldsymbol{I})$ and

$\boldsymbol{F}=f_{0} \boldsymbol{I}$.

For a uniaxial test to failure in tension, the yield criterion reads:

$F_{0} \sigma_{0}^{+}+f_{0} \sigma_{0}^{+}-1=0$

For a uniaxial test in compression, the criterion yields

$F_{0} \sigma_{0}^{-}-f_{0} \sigma_{0}^{-}-1=0$

By combining these equations, we find

$F_{0}=\frac{\sigma_{0}^{+}+\sigma_{0}^{-}}{2 \sigma_{0}^{+} \sigma_{0}^{-}}$

and

$f_{0}=\frac{1}{2}\left(\frac{1}{\sigma_{0}^{+}}-\frac{1}{\sigma_{0}^{-}}\right)$

The criterion has three independent parameters: $\sigma_{0}^{+}, \sigma_{0}^{-}$ and $\zeta_{0}$. The shear strength is given by

$\tau_{0}=\sqrt{\frac{1}{2 F_{0}^{2}\left(1+\zeta_{0}\right)}}$

For isotropic material symmetry, the stress space may be decomposed into two independent parts, shear and normal stress space. The second-order tensor $\boldsymbol{F}$ is proportional to identity. The tensor $\mathbb{F}$ may be decomposed in a hydrostatic and a deviatoric part by spectral decomposition, which means that one of its eigentensors is pointing in the direction of the hydrostatic axis. Therefore, the rank-1 correction $\mathbb{F}-\boldsymbol{F} \otimes \boldsymbol{F}$ corresponds to a translation in the direction of the dyad $\boldsymbol{I} \otimes \boldsymbol{I}$ together with an isotropic dilatation and does not lead to a rotation of the eigensystem irrespective of the parameters governing $\mathbb{F}$ and $\boldsymbol{F}$. The shape of the surface in the direction of the hydrostatic axis is determined by the interaction parameter $\zeta_{0}$. This may be seen in Fig. 1. Convexity of the quadric surface requires (5) and (6) to be fulfilled, which leads to the following conditions:

$\sigma_{0}^{ \pm} \geq 0, \quad \tau_{0} \geq 0, \quad-1 \leq \zeta_{0} \leq 0.5$.

After finding the convexity criterion, it is interesting to examine the criterion in normal stress space in the extreme cases $\zeta_{0}=0.5$ and $\zeta_{0}=-1$. Firstly, the solutions of the criterion on the trisectrix are determined by solving Eq. (11) for isotropic material symmetry and a hydrostatic stress state $S_{\text {hydro }}$. This yields the following result:

$\boldsymbol{S}_{\text {hydro }}^{ \pm}=\frac{\sigma_{0}^{-}\left(3 \sigma_{0}^{-} \sigma_{0}^{+}-3\left(\sigma_{0}^{-}\right)^{2} \pm \sqrt{\left.\left(\sigma_{0}^{+}\right)^{2}\left(\sigma_{0}^{-}+\sigma_{0}^{+}\right)^{2}\left(12-24 \zeta_{0}\right)\right)}\right.}{\left(\sigma_{0}^{-}+\sigma_{0}^{+}\right)^{2}\left(3+3 \zeta_{0}\right)-18 \sigma_{0}^{+} \sigma_{0}^{-}} \boldsymbol{I}$

In the case $\zeta_{0}=0.5$, the square root in the enumerator of (25) vanishes, that is, the two solutions of Eq. (25) degenerate 
to one. This means that the quadric yield surface takes a conical shape in this case as shown in Fig. 1. If $\zeta_{0}>0.5$, no real solution exists on the hydrostatic axis, confirming the requirements for convexity of the yield surface stated in (24). For $\zeta_{0}=-1$, the criterion (1) degenerates to

$\left|F_{0} \operatorname{tr} \boldsymbol{S}\right|-f_{0} \operatorname{tr} \boldsymbol{S}-1=0$

which describes two parallel planes defined by the uniaxial yield points in tension and compression, respectively (see Fig. 1).

In between those extreme values, the criterion takes an elliptical, paraboloidal or hyperboloidal shape. When $\zeta_{0}<$ 0.5 , more than one solutions exist. For $\zeta=\zeta_{0 \text {, crit }}$, the criterion takes the shape of a spheroidal paraboloid. For $\zeta_{0, \text { crit }}<\zeta_{0}<0.5$, it becomes one sheet of a hyperboloid because of (6). A prolate spheroid in normalized stress space is obtained for $0 \leq \zeta_{0}<\zeta_{0 \text {, crit }}$. For $-1<\zeta_{0}<0$, the criterion has the shape of an oblate spheroid. The variety of possible shapes in normal stress space are shown in Fig. 1.

In order to find the critical value $\zeta_{0 \text {, crit }}$ at which the yield surface changes its form from ellipsoidal to hyperboloidal, Eq. (15) is considered in the case of isotropy. Solving $\operatorname{Det}(\mathbb{F}-$ $\boldsymbol{F} \otimes \boldsymbol{F})\left.\right|_{\zeta_{0}=\zeta_{0, \text { crit }}}=0$ for $\zeta_{0, \text { crit }}$ provides the sought value of $\zeta_{0, \text { crit }}$ :

$\zeta_{0, \text { crit }}=\frac{-\left(\sigma_{0}^{-}\right)^{2}+4 \sigma_{0}^{-} \sigma_{0}^{+}-\left(\sigma_{0}^{+}\right)^{2}}{\left(\sigma_{0}^{+}+\sigma_{0}^{-}\right)^{2}}$.

In shear stress space, the isotropic criterion has the shape of a sphere with radius $\tau_{0}$ (Fig. 1 ).

\subsection{Cubic symmetry}

In the case of cubic symmetry, the tensors $\mathbb{F}$ and $\boldsymbol{F}$ defining (1) are given by

$$
\begin{aligned}
\mathbb{F}= & \sum_{i=1}^{3} F_{0}^{2} \boldsymbol{M}_{i} \otimes \boldsymbol{M}_{i}-\sum_{i, j=1 ; i \neq j}^{3} \zeta_{0} F_{0}^{2} \boldsymbol{M}_{i} \otimes \boldsymbol{M}_{j} \\
& +\sum_{i, j=1 ; i \neq j}^{3} \frac{F_{i j}^{2}}{2} \boldsymbol{M}_{i} \underline{\otimes} \boldsymbol{M}_{j}
\end{aligned}
$$

and

$\boldsymbol{F}=\sum_{i=1}^{3} f_{0} \boldsymbol{M}_{i}$

with

$M_{i}=\boldsymbol{m}_{i} \otimes \boldsymbol{m}_{i}$

defining the main axes of the cubic symmetry. In analogy to the isotropic case, we find:

$F_{0}=\frac{\sigma_{0}^{+}+\sigma_{0}^{-}}{2 \sigma_{0}^{+} \sigma_{0}^{-}}$ and

$f_{0}=\frac{1}{2}\left(\frac{1}{\sigma_{0}^{+}}-\frac{1}{\sigma_{0}^{-}}\right)$.

When a shear test is performed to failure with shear stresses acting on plane $i$ in direction $j$, the yield criterion in the case of cubic symmetry reads:

$F_{i j} \tau_{0}-1=0$.

Therefore, we get

$F_{i j}=\frac{1}{\tau_{0}}$.

The criterion has four independent parameters: $\sigma_{0}^{+}, \sigma_{0}^{-}$and $\zeta_{0}$ and $\tau_{0}$. The mathematical discussion of the criterion with cubic symmetry with respect to convexity and shape of the criterion yields the same results as in the isotropic case and will not be repeated at this point.

\subsection{Fabric-based orthotropy}

When modeling a material with an internal microstructure, it is of high importance to account for its morphology. Trabecular bone may be characterized by bone volume fraction $\rho$ and a fabric tensor $\boldsymbol{M}$ (Matsuura et al. 2008; Rincón-Kohli and Zysset 2009; Zysset and Rincón 2006) emerging from an approximation of its orientation distribution function by spherical harmonics (Kanatani 1984) or by an ellipsoid (Harrigan and Mann 1984). In the particular case of the ZyssetCurnier model (Zysset and Curnier 1995), the influence of these two factors on its homogenized mechanical properties is governed by two exponents $p$ and $q$ that need to be determined experimentally (Matsuura et al. 2008; Rincón-Kohli and Zysset 2009; Zysset and Rincón 2006; Wolfram et al. 2012). The number of independent material parameters for the fabric-based orthotropy is 5 in the case of an isotropic base material $\left(\sigma_{0}^{+}, \sigma_{0}^{-}, \chi_{0}, p\right.$ and $\left.q\right)$ or 6 in the case of cubic symmetry $\left(\sigma_{0}^{+}, \sigma_{0}^{-}, \chi_{0}, \tau_{0}, p\right.$ and $\left.q\right)$. Inhomogeneity and anisotropy of material properties may in this case be interpreted as a stretch of the original stress space with eigenvalues $m_{i}$ and eigenvectors $\boldsymbol{m}_{i}$ (Wolfram et al. 2012). The stress tensor can therefore be normalized with respect to fabric and density by an inversion of the stretch operation:

$\hat{\boldsymbol{S}}=\frac{\boldsymbol{M}^{-q} \boldsymbol{S} \boldsymbol{M}^{-q}}{\rho^{p}}$

with

$\boldsymbol{M}=\sum_{i=1}^{3} m_{i}\left(\boldsymbol{m}_{i} \otimes \boldsymbol{m}_{i}\right)$.

The $\boldsymbol{m}_{i}$ are the vectors describing the material orientation. $\boldsymbol{M}^{q}$ is determined analogously to (36) with the same 
eigenvectors $\boldsymbol{m}_{\boldsymbol{i}}$ and the eigenvalues $m_{i}^{q} . \boldsymbol{M}$ is symmetric $\left(\boldsymbol{M}=\boldsymbol{M}^{T}\right)$ and normalized so that

$\operatorname{tr} M=3$.

The criterion may then be rewritten in normalized stress space featuring at least cubic symmetry:

$Y(\hat{\boldsymbol{S}}):=\sqrt{\hat{\boldsymbol{S}}: \hat{\mathbb{F}} \hat{\boldsymbol{S}}}+\hat{\boldsymbol{F}}: \hat{\boldsymbol{S}}-1=0$.

The tensors $\hat{\mathbb{F}}$ and $\hat{\boldsymbol{F}}$ are given by

$$
\begin{aligned}
\hat{\mathbb{F}}= & \sum_{i=1}^{3} F_{0}^{2} \boldsymbol{M}_{i} \otimes \boldsymbol{M}_{i}-\sum_{i, j=1 ; i \neq j}^{3} \zeta_{0} F_{0}^{2} \boldsymbol{M}_{i} \otimes \boldsymbol{M}_{j} \\
& +\sum_{i, j=1 ; i \neq j}^{3} \frac{1}{2 \tau_{0}^{2}} \boldsymbol{M}_{i} \underline{\otimes} \boldsymbol{M}_{j}
\end{aligned}
$$

and

$\hat{\boldsymbol{F}}=\sum_{i=1}^{3} f_{0} \boldsymbol{M}_{i}$

The tensors $\mathbb{F}$ and $\boldsymbol{F}$ defining the criterion (1) in original stress space are obtained by:

$\mathbb{F}=\rho^{2 p}\left(\boldsymbol{M}^{q} \underline{\otimes} \boldsymbol{M}^{q}\right) \hat{\mathbb{F}}\left(\boldsymbol{M}^{q} \underline{\otimes} \boldsymbol{M}^{q}\right)$

$\boldsymbol{F}=\rho^{p}\left(\boldsymbol{M}^{q} \otimes \boldsymbol{M}^{q}\right) \hat{\boldsymbol{F}}=\rho^{p} \boldsymbol{M}^{q} \hat{\boldsymbol{F}} \boldsymbol{M}^{q}$.

Since the fabric tensor $\boldsymbol{M}$ is strictly positive definite, the discussion of the orthotropic fabric-based criterion in stress space is equivalent to the discussion of the cubic criterion in fabric-normalized stress space. The discussion with respect to convexity and shape of the criterion is then analogous to the isotropic case and is not repeated in detail here.

In the following paragraphs, some important aspects of the fabric-based orthotropic criterion in stress space will be discussed. In the case of orthotropic material symmetry, stress space may be divided into a normal and a shear stress space. The uniaxial strengths in the main material directions are given by

$\sigma_{i i}^{+}=\sigma_{0}^{+} \rho^{p} m_{i}^{2 q}, \quad \sigma_{i i}^{-}=\sigma_{0}^{-} \rho^{p} m_{i}^{2 q}$,

the interaction parameters of $\mathbb{F}$ and the shear strengths by

$\zeta_{i j}=\zeta_{0} \frac{m_{i}^{2 q}}{m_{j}^{2 q}}, \quad \tau_{i j}=\tau_{0} \rho^{p} m_{i}^{q} m_{j}^{q}$.

The solutions of the criterion in stress space collinear to $\boldsymbol{M}^{2 q}, \boldsymbol{S}_{\mathrm{ma}}^{ \pm}$, may be obtained by backtransformation of the hydrostatic solutions in normalized stress space $\hat{S}_{\text {hydro }}$, which are equivalent to the solutions presented in (25):

$\boldsymbol{S}_{\mathrm{ma}}^{ \pm}=\rho^{p} \boldsymbol{M}^{q} \hat{\boldsymbol{S}}_{\mathrm{hydro}}^{ \pm} \boldsymbol{M}^{q}$ (a) For $\zeta_{0}=0.5$, the two solutions $\boldsymbol{S}_{\mathrm{ma}}^{ \pm}$and $\boldsymbol{A}$ degenerate to one solution, the apex of the generalized cone. The coordinates of the apex may be obtained by:

$\boldsymbol{S}_{\text {apex }}=\left.\rho^{p} \boldsymbol{M}^{q} \hat{\boldsymbol{S}}_{\text {hydro }}\right|_{\zeta_{0}=0.5} \boldsymbol{M}^{q}$.

It lies on the plane $\boldsymbol{F}: S=1$ and therefore, according to requirement (6), the anisotropic generalized cone is a limiting case of the possible convex shapes of the yield function.

The only eigentensor of the dyad $\boldsymbol{F} \otimes \boldsymbol{F}$ is always collinear to $\boldsymbol{M}^{2 q}$ irrespective of the value of $\zeta_{0}$ as seen in (40) and (42). The eigensystem of $\mathbb{F}$, however, changes. For $\zeta_{0}=0.5$, one eigenvalue of $\mathbb{F}$ vanishes and the corresponding eigentensor is aligned with $\boldsymbol{M}^{2 q}$. Therefore, $\boldsymbol{M}^{2 q}$ is also an eigentensor of $\mathbb{F}-\boldsymbol{F} \otimes \boldsymbol{F}$ and determines the orientation of the hypercone in stress space.

(b) In the case of $\zeta_{0}=-1$, two eigenvalues of $\mathbb{F}$ with eigentensors in normal stress space vanish and $\boldsymbol{M}^{2 q}$ is an eigentensor of $\mathbb{F}$ with a positive eigenvalue. The criterion therefore degenerates to two parallel hyperplanes with the hypersurface normal $\boldsymbol{M}^{2 q}$ (see Fig. 2).

(c) For $\zeta_{0}=0$, however, the eigensystem of $\mathbb{F}$ is aligned with the material axes themselves. Therefore, the main orientation of the quadric surface rotates from $\boldsymbol{M}^{2 q}$ at $\zeta_{0}=0.5$ to a perpendicular direction for $\zeta_{0}=-1$. For $-1<\zeta_{0}<0.5$, the orientation is changing continuously with the parameter $\zeta_{0}$.

The midpoint of the equivalent quadratic form (11) may be determined using Eq. (14). If $\zeta_{0 \text {,crit }}<\zeta_{0}<0.5, \boldsymbol{A}$ lies outside of the convex surface, as the quadratic form (11) of the criterion from which the midpoint $\boldsymbol{A}$ is derived describes a hyperboloid of two sheets in this case.

The change of the shape, midpoint and main orientation of the criterion as a function of the parameter $\zeta_{0}$ in the case of fabric-based orthotropy is illustrated in Fig. 2 and the video file in the supplementary material of this article. The main orientation of the criterion is the eigentensor of $\mathbb{F}-\boldsymbol{F} \otimes \boldsymbol{F}$ with the largest eigenvalue.

\subsection{General orthotropy}

In the case of general orthotropy, the fourth-order tensor $\mathbb{F}$ is given by:

$$
\begin{aligned}
\mathbb{F}= & \sum_{i=1}^{3} F_{i i}^{2} \boldsymbol{M}_{i} \otimes \boldsymbol{M}_{i}-\sum_{i, j=1 ; i \neq j}^{3} \zeta_{i j} F_{i i}^{2} \boldsymbol{M}_{i} \otimes \boldsymbol{M}_{j} \\
& +\sum_{i, j=1 ; i \neq j}^{3} \frac{F_{i j}^{2}}{2} \boldsymbol{M}_{i} \underline{\otimes} \boldsymbol{M}_{j}
\end{aligned}
$$


Fig. 2 Midpoint, orientation and shape of the fabric-based orthotropic quadric yield surface in normal stress space for $\mathbf{a} \zeta_{0}=-1, \mathbf{b} \zeta_{0}=-0.5$, c $\zeta_{0}=0.0, \mathbf{d} 0<\zeta_{0}<\zeta_{0, \text { crit }}$, e $\zeta_{0, \text { crit }}<\zeta_{0}<0.5$, and f $\zeta_{0}=0.5$

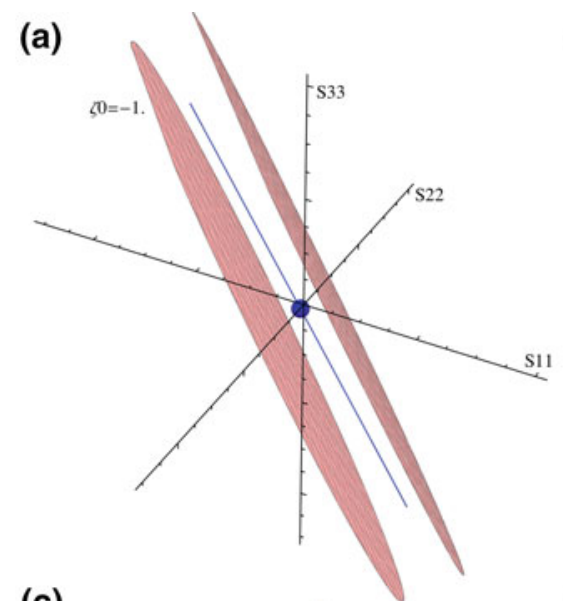

(c)

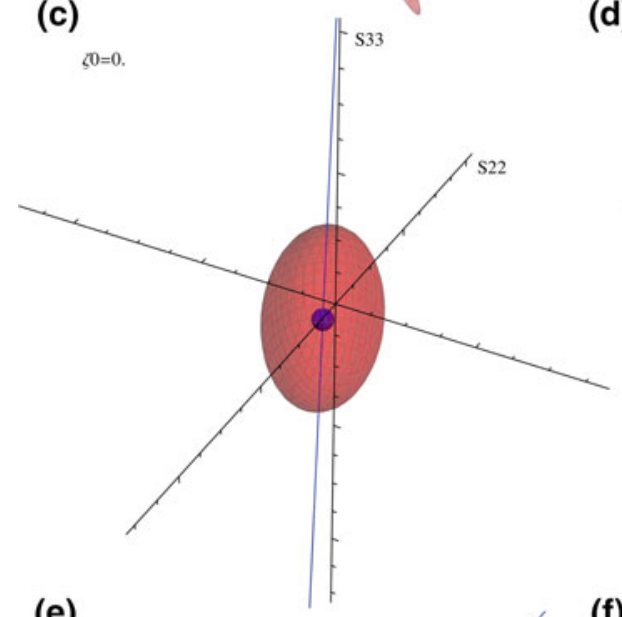

(e)

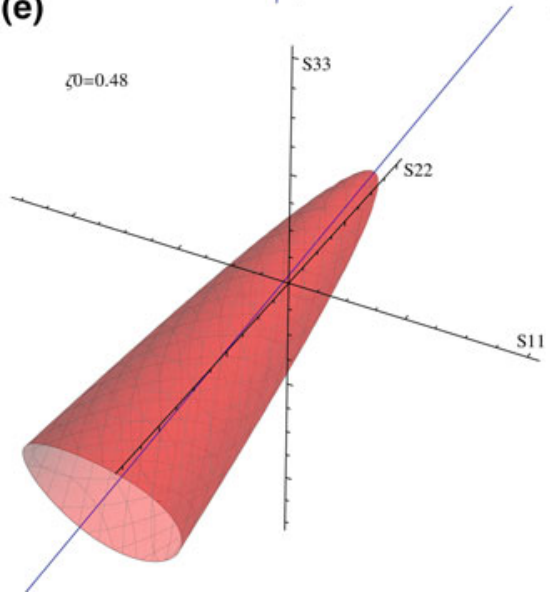

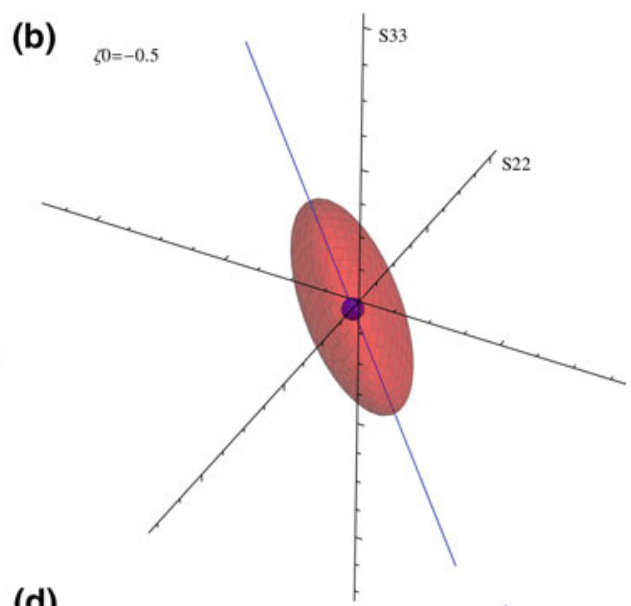

(d)

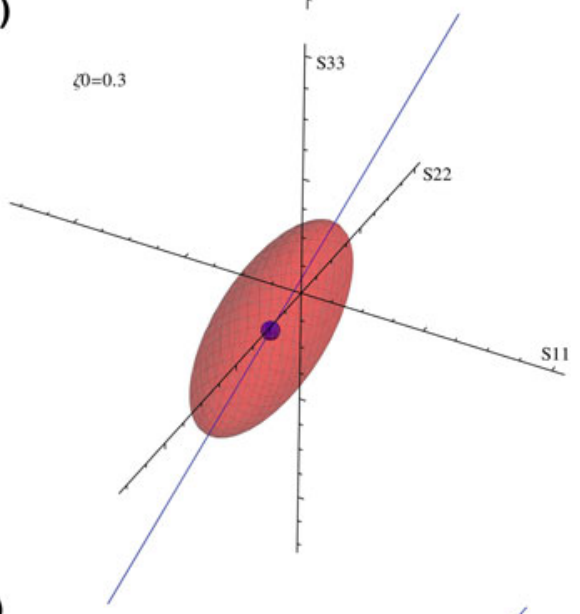

(f)

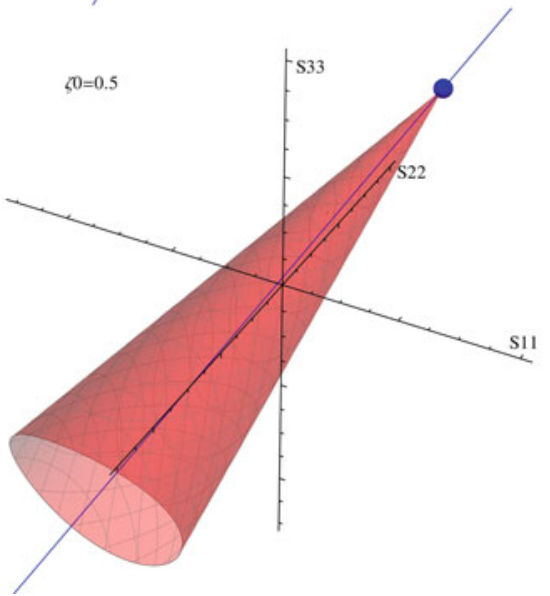

The general form of the second-order tensor $\boldsymbol{F}$ is in this case:

$\boldsymbol{F}=\sum_{i=1}^{3} f_{i} \boldsymbol{M}_{i}$

For a uniaxial test to failure in tension in the direction $i$, the yield criterion reads

$$
F_{i i} \sigma_{i i}^{+}+f_{i} \sigma_{i i}^{+}-1=0, \quad i=1,2,3
$$

For a uniaxial test in compression, the criterion yields

$F_{i i} \sigma_{i i}^{-}-f_{i} \sigma_{i i}^{-}-1=0, \quad i=1,2,3$.

By combining Eqs. (49) and (50), we find that

$$
F_{i i}=\frac{\sigma_{i i}^{+}+\sigma_{i i}^{-}}{2 \sigma_{i i}^{+} \sigma_{i i}^{-}}
$$

and

$f_{i}=\frac{1}{2}\left(\frac{1}{\sigma_{i i}^{+}}-\frac{1}{\sigma_{i i}^{-}}\right)$. 
When a shear test is performed to failure with shear stresses acting on plane $i$ in direction $j$, the yield criterion reads:

$F_{i j} \tau_{i j}-1=0$

Therefore, we get

$F_{i j}=\frac{1}{\tau_{i j}}$.

Twelve orthotropic constants are needed to define the criterion: the uniaxial yield stresses $\sigma_{11}^{+}, \sigma_{11}^{-}, \sigma_{22}^{+}, \sigma_{22}^{-}, \sigma_{33}^{+}$and $\sigma_{33}^{-}$, the shear yield stresses $\tau_{12}, \tau_{23}$ and $\tau_{13}$ as well as interaction coefficients $\zeta_{12}, \zeta_{23}$ and $\zeta_{13}$. For a homogeneous material, the criterion may be identified by a set of 6 uniaxial tests in the 3 main material directions in tension and compression providing the uniaxial yield stresses $\sigma_{i i}^{-}$and $\sigma_{i i}^{+}$. Three additional shear tests in 23,13 and 12 provide the shear yield stresses $\tau_{i j}$. The interaction parameters may be determined by independent biaxial tests in the 12,13 and 23 planes as discussed by Tsai and Wu (1971) or triaxial tests as done, for example, in silico by Wolfram et al. (2012). The requirements for convexity of the quadric are obtained from (5):

$\sigma_{i i}^{ \pm} \geq 0, \quad \tau_{i j} \geq 0, \quad\left|\zeta_{i j}\right| \leq\left|\frac{F_{j j}}{F_{i i}}\right|$

as well as

$$
\begin{aligned}
& F_{22}^{2} F_{33}^{2}-F_{11}^{2} F_{33}^{2} \zeta_{12}^{2}-F_{11}^{2} F_{22}^{2} \zeta_{13}^{2}+2 F_{11}^{2} F_{22}^{2} \zeta_{12} \zeta_{13} \zeta_{23} \\
& \quad-F_{22}^{4} \zeta_{23}^{2} \geq 0 .
\end{aligned}
$$

The transition from ellipsoidal to paraboloidal shape in normal stress space is taking place at any triplets $\left\{\zeta_{12}, \zeta_{13}, \zeta_{23}\right\}$ satisfying (15). In general, there is no analytical form for the roots of a cubic equation. However, a solution may be found numerically.

\section{Degeneration to classical yield criteria}

In the subsequent sections, the generality of formulation is demonstrated by showing its degeneration to several classical yield envelopes under appropriate conditions like the von Mises (1913); Prager and Drucker (1952); Tsai and Wu (1971); Liu et al. (1997), generalized Hill (Zysset and Rincón 2006) and classical (Hill 1951) criteria.

\subsection{Isotropic Drucker-Prager criterion}

When setting $\zeta_{0}=0.5$ in the isotropic case, the criterion (1) degenerates to

$F_{0} \sqrt{\frac{3}{2} \boldsymbol{S}: \mathbb{I}^{d} \boldsymbol{S}}+f_{0} \operatorname{tr} \boldsymbol{S}-1=0$ with the deviatoric tensor

$\mathbb{I}^{d}=\boldsymbol{I} \underline{\bar{\otimes}} \boldsymbol{I}-\frac{1}{3} \boldsymbol{I} \otimes \boldsymbol{I}$,

which describes a cone oriented in the direction of the hydrostatic axis and is identical to the criterion first proposed by Prager and Drucker (1952). In this case, the cohesion $h$ would be given by $h=\frac{1}{F_{0}}$ and the friction coefficient $T$ by $T=\frac{3 f_{0}}{F_{0}}$.

\subsection{Isotropic von Mises criterion}

When setting $\zeta_{0}=0.5$ and considering yield stresses that are symmetric around the origin $\left(\sigma_{0}^{+}=\sigma_{0}^{-}=\sigma_{0}\right)$ in the isotropic case, then $f_{0}=0$ and the second-order tensor $\boldsymbol{F}$ vanishes. In this case, the criterion (1) degenerates to

$\frac{1}{\sigma_{0}} \sqrt{\frac{3}{2} S: \mathbb{I}^{d} S}-1=0$,

which is the classical von Mises yield criterion (von Mises 1913).

\subsection{Orthotropic Tsai-Wu criterion}

When considering the equivalent quadratic form (11), there is an obvious similarity to the polynomial Tsai-Wu criterion (Tsai and Wu 1971; Cowin 1986; Wolfram et al. 2012), which has the form

$Y^{\mathrm{TW}}(\boldsymbol{S}):=\boldsymbol{S}: \mathbb{P} \boldsymbol{S}+\boldsymbol{P} \boldsymbol{S}-1=0$

with

$$
\begin{aligned}
\mathbb{P}= & \sum_{i=1}^{3} \frac{1}{\sigma_{i i}^{-} \sigma_{i i}^{+}} \boldsymbol{M}_{i} \otimes \boldsymbol{M}_{i}-\sum_{i, j=1 ; i \neq j}^{3} \frac{\chi_{i j}}{\sigma_{i i}^{-} \sigma_{i i}^{+}} \boldsymbol{M}_{i} \otimes \boldsymbol{M}_{j} \\
& +\sum_{i, j=1 ; i \neq j}^{3} \frac{1}{2 \tau_{i j}^{2}} \boldsymbol{M}_{i} \underline{\otimes} \boldsymbol{M}_{j}
\end{aligned}
$$

and

$$
\boldsymbol{P}=\sum_{i=1}^{3}\left(\frac{1}{\sigma_{i i}^{+}}-\frac{1}{\sigma_{i i}^{-}}\right) \boldsymbol{M}_{i} .
$$

Comparison of the two criteria shows that a straightforward transformation exists from the Tsai-Wu to the quadric criterion. For known tensors $\mathbb{P}$ and $\boldsymbol{P}$, the tensors $\mathbb{F}$ and $\boldsymbol{F}$ defining the quadric surface are given by:

$$
\begin{aligned}
& \boldsymbol{F}=\frac{\boldsymbol{P}}{2} \\
& \mathbb{F}=\mathbb{P}+\frac{\boldsymbol{P} \otimes \boldsymbol{P}}{4}
\end{aligned}
$$

The 6 uniaxial and 3 shear yield stresses are not altered by this transformation. Element-wise comparison of the tensors $\mathbb{P}$ and $(\mathbb{F}-\boldsymbol{F} \otimes \boldsymbol{F})$ shows that the interaction parameters $\zeta_{i j}$ 
of the quadric criterion may be determined from the known $\chi_{i j}$ of the Tsai-Wu criterion by:

$\zeta_{i j}=\frac{1}{F_{i i}^{2}}\left(\frac{\chi_{i j}}{\sigma_{i i}^{+} \sigma_{i i}^{-}}-f_{i} f_{j}\right)$

\subsection{Orthotropic Liu criterion}

If the interaction coefficients $\zeta_{i j}$ are determined by a specific coupling:

$$
\begin{gathered}
\zeta_{i j}=\frac{1}{2 F_{i i}^{2}}\left(F_{i i}^{2}+F_{j j}^{2}-F_{k k}^{2}\right) \quad \forall i, j, k \in \mathcal{N}, \\
i, j, k \leq 3, \quad i \neq j \neq k,
\end{gathered}
$$

where no summation should be performed on repeated indices, Liu's criterion (Liu et al. 1997) is obtained, which is a generalization of the Drucker-Prager criterion to plastically orthotropic materials, that is, a generalized cone in normal stress space aligned with the hydrostatic axis.

\subsection{Orthotropic Hill criterion}

If the yield stresses are symmetric around the origin $\left(\sigma_{i}^{+}=\right.$ $\sigma_{i}^{-}$), the second-order tensor $\boldsymbol{F}$ vanishes and (1) degenerates to

$\sqrt{S: \mathbb{F} S}-1=0$,

which is a generalized Hill criterion as described by, for example, (Zysset and Rincón 2006). After transformation of the interaction coefficients $\zeta_{i j}$ according to (66), Eq. (67) describes the classical Hill criterion for plastically orthotropic materials (Hill 1951), which is a generalized cylinder in normal stress space aligned with the hydrostatic axis.

\section{Application to a micromechanical yield criterion for porous solids}

In the following section, the applicability of the yield criterion (1) to micromechanical analyses will be demonstrated by identifying a quadric criterion based of an existing micromechanics-based formulation. Maghous et al. (2009) proposed a micromechanical yield criterion for isotropic porous cohesive-frictional solids based on nonlinear homogenization techniques. The matrix material is governed by a Drucker-Prager yield surface with cohesion $h$ and friction coefficient $T, \phi$ denotes the pore volume fraction. This criterion is to some extend applicable to bone, which may be described at the microscale as a cohesive-frictional material (Tai et al. 2006) interacting with nanoporosity. It has the form (Maghous et al. 2009):

$$
\begin{aligned}
Y_{p}(\boldsymbol{S}, \phi)= & \frac{1+\frac{2}{3} \phi}{T^{2}} \Sigma_{d}^{2}+\left(\frac{3 \phi}{2 T^{2}}-1\right) \Sigma_{m}^{2} \\
& +2(1-\phi) h \Sigma_{m}-(1-\phi)^{2} h^{2}=0,
\end{aligned}
$$

with

$\Sigma_{m}=\frac{\operatorname{tr} \boldsymbol{S}}{3}, \quad \Sigma_{d}=\sqrt{\left(\boldsymbol{S}-\Sigma_{m} \boldsymbol{I}\right):\left(\boldsymbol{S}-\Sigma_{m} \boldsymbol{I}\right)}$,

and predicts an elliptical criterion above a critical porosity $\phi_{\text {crit }}=\frac{4 T^{2}}{3}$. Below the critical porosity, the criterion describes a hyperboloid or a double-cone and is nonconvex. It may be rewritten in the following form:

$$
\begin{aligned}
& \boldsymbol{S}:\left(\frac{1+\frac{2}{3} \phi}{(1-\phi)^{2} h^{2} T^{2}} \mathbb{I}^{d}+\frac{\frac{3 \phi}{2 T^{2}}-1}{9(1-\phi)^{2} h^{2}} \boldsymbol{I} \otimes \boldsymbol{I}\right) \boldsymbol{S} \\
& \quad+\frac{2}{3(1-\phi) h} \boldsymbol{I}: \boldsymbol{S}-1=0 .
\end{aligned}
$$

This is a quadratic polynomial in $S$, and therefore the isotropic tensors $\mathbb{F}_{p}$ and $\boldsymbol{F}_{p}$ defining (1) may be determined by comparison with the quadratic form (11). They are:

$\mathbb{F}_{p}=\frac{1+\frac{2}{3} \phi}{(1-\phi)^{2} h^{2} T^{2}}(\boldsymbol{I} \underline{\bar{\otimes}} \boldsymbol{I})-\frac{\frac{1}{3}+\frac{1}{18} \phi}{(1-\phi)^{2} h^{2} T^{2}}(\boldsymbol{I} \otimes \boldsymbol{I})$

and

$\boldsymbol{F}_{p}=\frac{1}{3(1-\phi) h} \boldsymbol{I}$.

The criterion rewritten in the form of (1) then predicts the same elliptical criteria for overcritical porosities and a conical one for the solid matrix. However, it predicts convex (half) hyperboloidal criteria for undercritical porosities, which extends the validity of the existing micromechanical formulation as presented by Maghous et al. (2009) as a yield criterion to undercritical states of pore volume fraction. Thus, a smooth transition is found from a conical criterion for the solid matrix to elliptical criteria for $\phi>\phi_{\text {crit }}$ through convex (half) hyperboloids. This is illustrated in Fig. 3.

\section{Identification of yield criteria for bone}

In the following section, the advantage in using a very general formulation like the one proposed in this work when describing a hierarchical material like bone will be demonstrated by describing the varying yield envelopes of bone at several length scales. Elliptical criteria describing the yield envelope of homogenized trabecular bone at the macroscale from experimental data and numerical homogenization techniques as well as a conical criterion for lamellar bone at the microscale will be identified. 


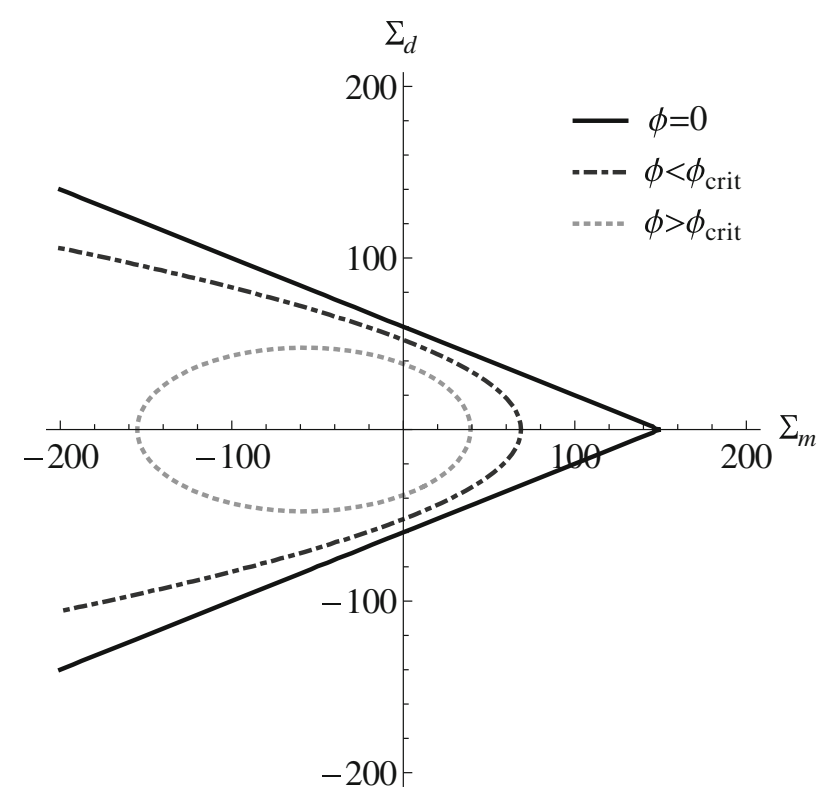

Fig. 3 Quadric yield surface for isotropic porous cohesive-frictional materials (Maghous et al. 2009) for friction coefficient $T=0.4$ and cohesion $h=150 \mathrm{MPa}$ as a function of pore volume fraction $\phi$ in the $\Sigma_{d}-\Sigma_{m}$ plane showing a smooth transition from conical to elliptical surfaces

\subsection{Trabecular bone}

A fabric- and density-based orthotropic quadric yield and strength criterion for trabecular bone was fitted to the multiaxial experimental data obtained by Rincón-Kohli and Zysset (2009). In this study, a multiaxial loading chamber was used to determine the compressive multiaxial yield and strength properties of cancellous bone from several anatomical locations. Cylindrical cancellous bone samples were dissected from fresh frozen bones of 12 donors ( 5 males and 7 females) with a mean age of 73.5 years (30-89 years). Their morphology was assessed by means of $\mu \mathrm{CT}$ scans. Then, different mechanical tests were performed such as torsion, uni-axial traction, uni-axial compression and multiaxial compression. A total of 128 bone samples were tested to failure. The yield point was determined according to the $0.2 \%$ offset criterion, strength was defined as ultimate force before failure divided by mean area. A fabric- and density-based quadric criterion was then fitted to the experimental data by means of the optimization routine Nminimize (Mathematica, Wolfram Research Inc.). The resulting constants for trabecular bone as well as the number of observations $(N)$, standard error of the estimate (SEE) and $R^{2}$ are shown in Tables 1 and 2 for the yield and strength criteria, respectively.

The quadric strength criterion is illustrated in normalized stress space in Fig. 4.

It is worth noting that even though the yield and strength criteria have a similar shape in both the normal and the shear
Table 1 Tensile yield stress $\sigma_{0}^{+}$, compressive yield stress $\sigma_{0}^{-}$, interaction parameter $\zeta_{0}$, shear yield stress $\tau_{0}$, exponents $p$ and $q$ as well as number of load cases $N$, standard error of the estimate SEE and $R^{2}$ of a yield criterion for trabecular bone in MPa obtained from experimental data (Rincón-Kohli and Zysset 2009)

\begin{tabular}{lllllllll}
\hline$\sigma_{0}^{+}$ & $\sigma_{0}^{-}$ & $\zeta_{0}$ & $\tau_{0}$ & $p$ & $q$ & $N$ & SEE & $R^{2}$ \\
\hline 32.7 & 47.8 & 0.220 & 11.1 & 1.28 & 0.503 & 110 & 0.26 & 0.94
\end{tabular}

Table 2 Tensile strength $\sigma_{0}^{u+}$, compressive strength $\sigma_{0}^{u-}$, interaction parameter $\zeta_{0}^{u}$, shear strength $\tau_{0}^{u}$, exponents $p^{u}$ and $q^{u}$ as well as number of load cases $N$, standard error of the estimate SEE and $R^{2}$ of a strength criterion for trabecular bone in MPa obtained from experimental data (Rincón-Kohli and Zysset 2009)

\begin{tabular}{lllllllll}
\hline$\sigma_{0}^{u+}$ & $\sigma_{0}^{u-}$ & $\zeta_{0}^{u}$ & $\tau_{0}^{u}$ & $p^{u}$ & $q^{u}$ & $N$ & SEE & $R^{2}$ \\
\hline 39.7 & 53.2 & 0.226 & 22.9 & 1.29 & 0.593 & 95 & 0.259 & 0.933 \\
\hline
\end{tabular}

stress space, the strength criterion is not an isotropic expansion of the yield criterion as defined in Eq. (3). It may, however, be described by an expansion of the yield criterion with distinct hardening ratios $r_{n}=1.1$ in normal and $r_{s}=2.1$ in shear stress space. This might be accomplished by using the yield data presented in Table 1 in a criterion with a tensorial hardening rule as introduced in Eq. (4) with a tensor function $\mathbb{K}(\kappa)$ of the form

$$
\begin{aligned}
\mathbb{K}(\kappa)= & \sum_{i=1}^{3} \frac{\boldsymbol{M}_{i} \otimes \boldsymbol{M}_{i}}{1+\left(r_{n}-1\right) f_{n}(\kappa)} \\
& +\sum_{i, j=1 ; i \neq j}^{3} \frac{\boldsymbol{M}_{i} \underline{\otimes} \boldsymbol{M}_{j}}{1+\left(r_{s}-1\right) f_{s}(\kappa)},
\end{aligned}
$$

where $f_{n}(\kappa)$ and $f_{s}(\kappa)$ are appropriate scalar hardening evolution functions bounded by $0 \leq f_{n}(\kappa), f_{s}(\kappa) \leq 1$ and the $\boldsymbol{M}_{i}$ are defined by (30). This would allow to describe both the experimental yield and strength data by one criterion.

Alternatively, a fabric- and density-based orthotropic yield criterion for trabecular bone was obtained by conversion of the Tsai-Wu criterion proposed by Wolfram et al. (2012). In this particular study, nonlinear $\mu \mathrm{FE}$ models with cancellous bone cubes of 5.62-mm edge length were generated from $\mu \mathrm{CT}$ scans. Kinematic boundary conditions were imposed testing the specimen in force control in 17 different load cases (six uniaxial, three shear and eight multiaxial) beyond yield. The yield point was determined according to the $0.2 \%$ offset rule. The study included 16 fresh frozen vertebrae (T6 to L2) from 10 donors ( 7 males and 3 females) with a median age of 51 years (37-84 years). The fabric-and density-based Tsai-Wu criterion was fitted to the resulting yield data using the optimization routine Nminimize (Mathematica, Wolfram Research Inc.). The interaction parameters were then transformed using Eq. (65). The resulting constants of the quadric 
Fig. 4 Quadric strength surface for trabecular bone and experimental data

(Rincón-Kohli and Zysset 2009) in a normalized normal stress space and $\mathbf{b}$ the $\hat{S}_{33}-\hat{S}_{12}$ plane

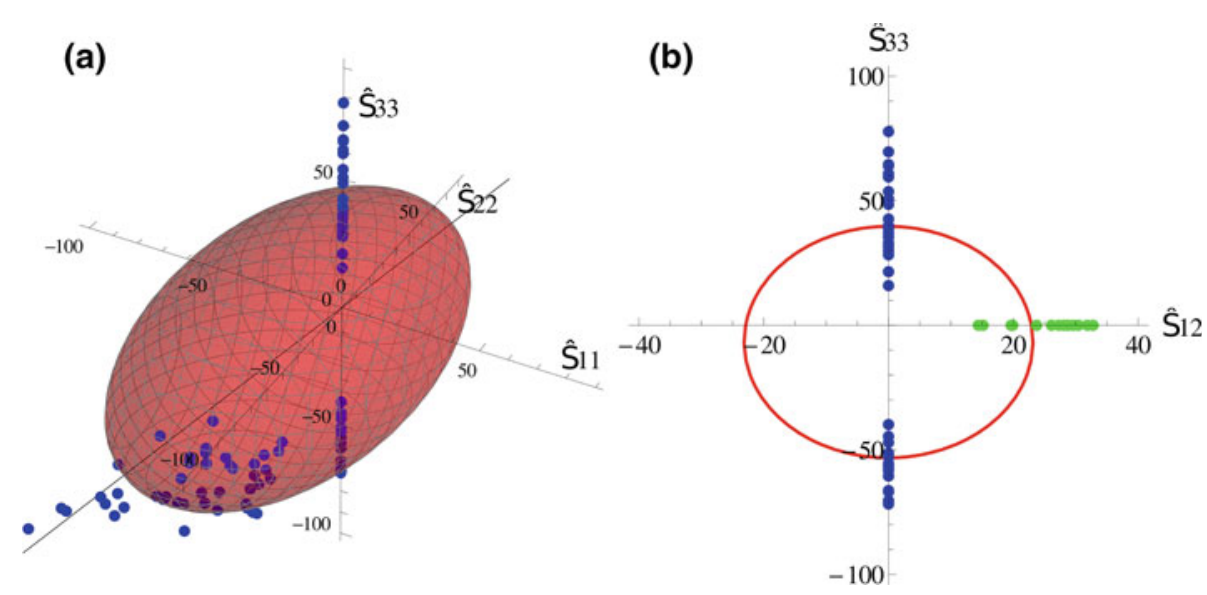

Table 3 Tensile yield stress $\sigma_{0}^{+}$, compressive yield stress $\sigma_{0}^{-}$, interaction parameter $\zeta_{0}$, shear yield stress $\tau_{0}$, exponents $p$ and $q$ as well as number of load cases $N$, standard error of the estimate SEE and $R^{2}$ of a yield criterion for trabecular bone in MPa obtained by numerical homogenization techniques (Wolfram et al. 2012)

\begin{tabular}{lllllllll}
\hline$\sigma_{0}^{+}$ & $\sigma_{0}^{-}$ & $\zeta_{0}$ & $\tau_{0}$ & $p$ & $q$ & $N$ & SEE & $R^{2}$ \\
\hline 74.589 & 111.724 & 0.2182 & 47.3314 & 1.686 & 1.02 & 391 & 0.1358 & 0.93
\end{tabular}

yield criterion for trabecular bone as well as $N$, SEE and $R^{2}$ are given in Table 3.

The quadric yield criterion for trabecular bone is shown in normalized stress space in Fig. 5.

\subsection{Lamellar bone}

It has been proposed that lamellar bone behaves as a cohesivefrictional material at the nanoscale due to its nanogranular structure (Tai et al. 2006; Wang et al. 2008; Carnelli et al. 2010). Here, the parameters of the quadric yield surface for bone tissue at the nanoscale were identified based on the work presented by Carnelli et al. (2010, 2011). In this particular study, transversely isotropic yield properties of bovine bone tissue were determined by means of a combination of nanoindentation experiments in axial and transverse direction with a numerical sensitivity study. Carnelli et al. (2011) reported an axial compressive yield strength $\sigma_{33}^{-}=150 \mathrm{MPa}$, a transverse to axial ratio $R_{\mathrm{TA}}=0.86$ and a cone opening angle $\beta=25^{\circ}$ of an underlying fictitious isotropic material. Using the relations $\sigma_{i i}^{-}=\sigma_{0}^{-} \rho^{p} m_{i}^{2 q}, R_{\mathrm{TA}}=\frac{m_{1}^{2 q}}{m_{3}^{2 q}}, m_{1}=$ $m_{2}=\frac{3-m_{3}}{2}$ for transverse isotropy, $\tan \beta=\frac{3 f_{0}}{F_{0}}$ and (23), and setting $p=0.0, q=1.0$, as well as $\zeta_{0}=0.5$, the yield constants provided by Carnelli et al. (2011) were converted to a fabric-based quadric criterion. The resulting properties are shown in Table 4.
The yield surface for lamellar bone is presented in Fig. 6 .

\section{Discussion}

In this work, a generalized anisotropic quadric yield criterion was proposed and discussed in stress space. The convexity limits of the criterion and the transition points between the different shapes were identified. The criterion was discussed for special cases of material symmetry such as isotropy, cubic symmetry, fabric-based orthotropy and general orthotropy. It was shown that the criterion degenerates to several classical yield criteria under appropriate conditions like the ones proposed by von Mises (1913), Prager and Drucker (1952), Tsai and Wu (1971), Liu et al. (1997), generalized Hill (Zysset and Rincón 2006) and classical Hill (1951) criteria. Applicability of the formulation for micromechanical analyses was shown by the identification of a quadric yield surface from a micromechanics-based criterion for porous cohesivefrictional materials (Maghous et al. 2009). This is particularly interesting for the description of bone, as it may be described on the micro-level as a cohesive-frictional material (Tai et al. 2006) interacting with nanoporosity. It was shown that the formulation as a quadric criterion extends the convexity of the original criterion (Maghous et al. 2009) to undercritical porosities. Thus, a smooth transition from the conical yield surface of the solid matrix material to an elliptical criterion of the porous composite is found. Also, a fabric- and density-based quadric strength criterion was identified for trabecular bone from multiaxial experimental data of Rincón-Kohli and Zysset (2009). A tensorial hardening rule was proposed allowing to fit both the experimental yield and strength data of Rincón-Kohli and Zysset (2009) by a single criterion. Additionally, the fabric- and densitybased Tsai-Wu yield criterion for trabecular bone identified by Wolfram et al. (2012) from in silico data was converted to an equivalent quadric criterion by a transformation of the 
Fig. 5 Quadric yield surface for trabecular bone and in silico data (Wolfram et al. 2012) in normalized a normal and $\mathbf{b}$ shear stress space
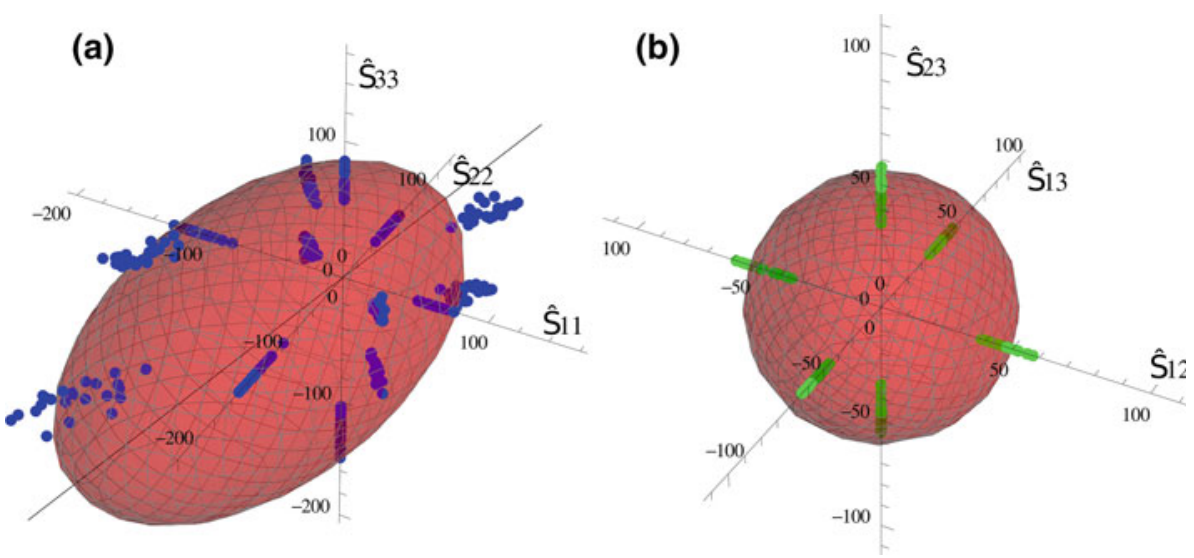

Table 4 Tensile yield stress $\sigma_{0}^{+}$, compressive yield stress $\sigma_{0}^{-}$, interaction parameter $\zeta_{0}$, shear yield stress $\tau_{0}$, exponents $p$ and $q$ as well as the eigenvalues of the fabric tensor $m 1, m 2$ and $m 3$ of a yield criterion for bovine lamellar bone in MPa from a nanoindentation study (Carnelli et al. 2011)

\begin{tabular}{llllllll}
\hline$\sigma_{0}^{+}$ & $\sigma_{0}^{-}$ & $\zeta_{0}$ & $\tau_{0}$ & $p$ & $q$ & $m 1=m 2$ & $m 3$ \\
\hline 100 & 136 & 0.5 & 66.6 & 0.0 & 1.0 & 0.975 & 1.05 \\
\hline
\end{tabular}

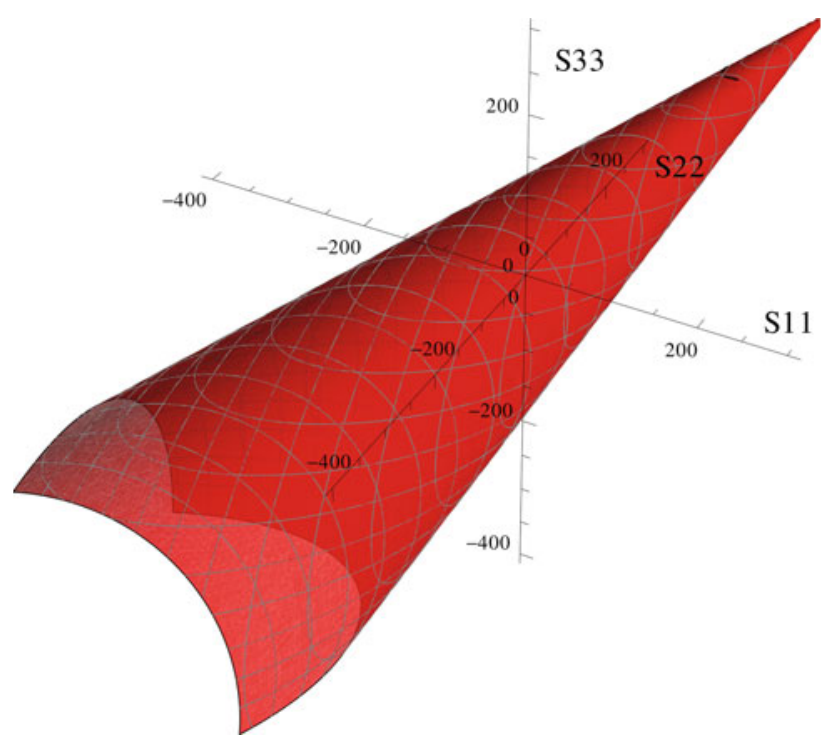

Fig. 6 Conical yield surface for lamellar bone from nanoindentation study (Carnelli et al. 2011) in normal stress space

interaction parameter. Finally, a conical yield criterion for bovine lamellar bone was identified from a nanoindentation study by Carnelli et al. (2011).

Bone features a varying amount of porosity on every hierarchical level and a nanogranular ultrastructure. Therefore, the quadric yield criterion with its ability to take different convex quadric shapes is suitable to approximate the yield envelope at every hierarchical level starting from the extracellular matrix on the microscale to the macroscopic bone tissue.
The formulation allows material symmetries ranging from isotropy to general anisotropy. It may take different convex quadratic shapes with a smooth transition from ellipsoidal to conical or cylindrical ones, making it a suitable candidate for use with a wide range of different materials such as metals, geomaterials, porous foams and bone. The generality of the formulation is also beneficial in the case of material identification. If the shape of the yield function is not known a priori, a minimization using the quadric criterion will result in the optimal shape among the convex quadrics eliminating the need for several trial identifications using different formulations. It might prove especially interesting in combination with multiscale homogenization techniques in the future, as it may be used for the homogenized material description at several length scales as long as the failure envelope of the material can be approximated by a quadric surface.

The proposed criterion is a homogeneous function of degree one, which is beneficial for the implementation of isotropic hardening rules in a computational mechanics framework. For isotropic hardening (3), its gradients with respect to stress and the hardening variable are independent of each other, thus simplifying the implementation of, for example, backprojection algorithms. Also, it allows to make conservative approximations of the elastic domain of cohesive-frictional materials governed by a Drucker-Prager-type conical yield surface by a smooth hyperboloid. This solves the common problem of nonuniqueness of the normal of the surface at the cone apex and allows the use of standard backprojection algorithms. The quadric criterion proved to be an effective tool in the description of bone strength at several length scales. Due to its generality and simple structure, it is well suited for the use in computational inelasticity of materials and structures.

Acknowledgments This work was supported by the University of Bern and a Ph.D. scholarship of the German National Academic Foundation. 


\section{References}

Arramon YP, Mehrabadi MM, Martin DW, Cowin SC (2000) A multidimensional anisotropic strength criterion based on Kelvin modes. Int J Solids Struct 37:2915-2935

Bayraktar HH, Gupta A, Kwon RY, Papadopoulos P, Keaveny TM (2004) The modified super-ellipsoid yield criterion for human trabecular bone. J Biomech Eng 6:677-684

Carnelli D, Gastaldi D, Sassi V, Contro R, Ortiz C, Vena P (2010) A finite element model for direction-dependent mechanical response to nanoindentation of cortical bone allowing for anisotropic post-yield behavior of the tissue. J Biomech Eng 132(8):081008

Carnelli D, Lucchini R, Ponzoni M, Contro R, Vena P (2011) Nanoindentation testing and finite element simulations of cortical bone allowing for anisotropic elastic and inelastic mechanical response. J Biomech 44(10):1852-1858

Cowin SC (1979) On the strength anisotropy of bone and wood. J Appl Mech 46(4):832-838

Cowin SC (1985) The relationship between the elasticity tensor and the fabric tensor. Mech Mater 4(2):137-147

Cowin SC (1986) Fabric dependence of an anisotropic strength criterion. Mech Mater 5:251-260

Cowin SC (1989) Bone mechanics. CRC press, Boca Raton, FL

Cowin SC, He QC (2005) Tensile and compressive stress yield criteria for cancellous bone. J Biomech 38(1):141-144

Curnier A (1994) Computational methods in solid mechanics, vol 29. Springer, Berlin

Drucker D, Prager W (1952) Soil mechanics and plastic analysis or limit design. Q Appl Math 10:157

Fratzl P, Weinkamer R (2007) Nature's hierarchical materials. Prog Mater Sci 52(8):1263-1334

Gelfand IM, Kapranov MM, Z A (1994) Discriminants, resultants and multidimensional determinants. Birkhuser, Boston

Gibson L (1985) The mechanical behaviour of cancellous bone. J Biomech 18(5):317-328

Gupta H, Zioupos P (2008) Fracture of bone tissue: the 'hows' and the 'whys'. Med Eng Phys 30(10):1209-1226

Harrigan TP, Mann RW (1984) Characterisation of microstructural anisotropy in orthotropic materials using a second rank tensor. J Mater Sci 19:761-767

Hellmich C, Ulm F-J (2002) Are mineralized tissues open crystal foams reinforced by crosslinked collagen? Some energy arguments. J Biomech 35(9):1199-1212

Hildebrand T, Laib A, Müller R, Dequeker J, Rüegsegger P (1999) Direct three-dimensional morphometric analysis of human cancellous bone: microstructural data from spine, femur, iliac crest, and calcaneus. J Bone Miner Res 14(7):1167-1174

Hill R (1951) The mathematical theory of plasticity. Oxford University Press, Oxford

Kanatani K-I (1984) Distribution of directional data and fabric tensors. Int J Eng Sci 22(2):149-164

Keaveny TM, Morgan EF, Niebur GL, Yeh OC (2001) Biomechanics of trabecular bone. Annu Rev Biomed Eng 3(1):307-333

Kristic R (1991) Human microscopic anatomy. Springer, Berlin

Liu C, Huang Y, Stout M (1997) On the asymmetric yield surface of plastically orthotropic materials: a phenomenological study. Acta Mater 45(6):2397-2406

Maghous S, Dormieux L, Barthélémy JF (2009) Micromechanical approach to the strength properties of frictional geomaterials. Eur J Mech A 28(1):179-188
Matsuura M, Eckstein F, Lochmüller E-M, Zysset P (2008) The role of fabric in the quasi-static compressive mechanical properties of human trabecular bone from various anatomical locations. Biomech Model Mechanobiol 7:27-42

Mehrabadi M, Cowin S (1990) Eigentensors of linear anisotropic elastic materials. Q J Mech Appl Math 43(1):15-41

Parfitt A (1984) Age-related structural changes in trabecular and cortical bone: cellular mechanisms and biomechanical consequences. Calcif Tissue Int 36:S123-S128

Prager W, Drucker D (1952) Soil mechanics and plastic analysis or limit design, 0. Appi Math 10(2):157-165

Reisinger A, Pahr D, Zysset PK (2010) Elastic anisotropy of bone lamellae as a function of fibril orientation pattern. Biomech Model Mechanobiol 10(1):67-77

Rho JY, Kuhn-Spearing L, Zioupos P (1998) Mechanical properties and the hierarchical structure of bone. Med Eng Phys 20(2):92-102

Rincón-Kohli L, Zysset P (2009) Multi-axial mechanical properties of human trabecular bone. Biomech Model Mechanobiol 8:195-208

Shih CF, Lee D (1978) Further developments in anisotropic plasticity. J Eng Mater 100(3):294-302

Smith CI, Faraldos M, Fernández-Jalvo Y (2008) The precision of porosity measurements: effects of sample pre-treatment on porosity measurements of modern and archaeological bone. Palaeogeogr Palaeoclimatol Palaeoecol 266:175-182

Tai K, Ulm F-J, Ortiz C (2006) Nanogranular origins of the strength of bone. Nano Lett 6(11):2520-2525

Tsai S, Wu E (1971) A general theory of strength for anisotropic materials. J Compos Mater 5(1):58-80

von Mises R (1913) Mechanik der festen Körper im plastisch deformablen Zustand. Göttin Nachr Math Phys 1:582-592

Wang R, Gupta HS (2011) Deformation and fracture mechanisms of bone and nacre. Annu Rev Mater Res 41:41-73

Wang X, Allen MR, Burr DB, Lavernia EJ, Jeremic B, Fyhrie DP (2008) Identification of material parameters based on Mohr-Coulomb failure criterion for bisphosphonate treated canine vertebral cancellous bone. Bone 43(4):775-780

Weiner S, Wagner HD (1998) The material bone: structure-mechanical function relations. Annu Rev Mater Sci 28(1):271-298

Whitehouse W (1974) The quantitative morphology of anisotropic trabecular bone. J Microsc 101:153-168

Wolfram U, Gross T, Pahr D, Schwiedrzik JJ, Wilke H-J, Zysset PK (2012) Fabric based Tsai-Wu yield criteria for vertebral trabecular bone in stress and strain space. J Mech Behav Biomed 15:218-228

Yeni Y, Dong X, Fyhrie D, Les C (2004) The dependence between the strength and stiffness of cancellous and cortical bone tissue for tension and compression: extension of a unifying principle. Biomed Mater Eng 14(3):303-310

Zysset PK (2003) A review of morphology-elasticity relationships in human trabecular bone: theories and experiments. J Biomech 36:1469-1485

Zysset PK, Curnier A (1995) An alternative model for anisotropic elasticity based on fabric tensors. Mech Mater 21(4):243-250

Zysset PH, Rincón L (2006) An alternative fabric-based yield and failure criterion for trabecular bone. In: Holzapfel GA, Ogden RW (eds) Mechanics of biological tissue, Springer, pp 457-470 